\title{
THEORY OF MEROMORPHIC FUNCTIONS ON AN OPEN RIEMANN SURFACE WITH NULL BOUNDARY
}

\author{
MASATSUGU TSUJI
}

In the former paper, ${ }^{11}$ I have developped a theory of meromorphic functions in a neighbourhood of a bounded closed set $E$ of logarithmic capacity zero, by means of Evans' potential fnnction $u(z)$, which tends to $\infty$, when $z$ tends to any point of $E$. It is not krown, whether such a potential function exists on ari open Riemann surface with null boundary, but by a substitute of Evans' function, we shall develop the similar theory of meromorphic functions on an open Riemann surface with null boundary.

\section{$\S 1$}

1. Let $F$ be an open Riemann surface with null boundary, spread over the $z$-plane. We exhaust $F$ by a sequence of compact Riemann surfaces: $F_{0} \subset F_{1} \subset \ldots \subset F_{n} \rightarrow F$, where the boundary $I_{n}$ of $F_{n}$ consists of a finite number of analytic Jordan curves.

Let $u_{n}(z)$ be the harmonic measure of $\Gamma_{n}$ with respect to $F_{n}-\bar{F}_{0}$, such that $u_{n}(z)$ is harmonic in $F_{n}-\bar{F}_{0}, u_{n}(z)=0$ on $\Gamma_{0}, u_{n}(z)=1$ on $\Gamma_{n}$. Then as well known, $\lim _{n \rightarrow \infty} u_{n}(z)=0$ uniformly in any compact domain of $F$. Let $v_{n}(z)$ be the conjugate harmonic function of $u_{n}(z)$ and

$$
d_{n}=\int_{\Gamma_{0}} d v_{n}(z)
$$

then

$$
d_{1} \geqq d_{2} \geq \ldots \geq d_{n} \rightarrow 0 \text {. }
$$

We put

$$
\zeta=e^{\frac{2 \pi}{d_{n}\left(u_{n}(z)+i v_{n}(z)\right)}}=r e^{i \theta},
$$

where

$$
r=r_{n}(z)=e^{\frac{2 \pi}{d_{n}\left(x_{n, 2}\right)}}, \quad \theta=\theta_{n}(z)=\frac{2 \pi}{d_{n}} v_{n}(z)
$$

then

$$
1 \leqq r \leqq r_{n}, \quad r_{n}=e^{\frac{2 \pi}{d_{n}}}
$$

Received July 31, 1953.

1) M. Tsuji: On the behaviour of a mernmorphic function in the neighbourhood of a closed set of capacity zero. Proc. Imp. Acad. 18 (1942). M. Tsuji: Theory of meromorphic functions in an neighbourhood of a closed set of capacity zero. Jap. Journ. Math. 19 (1944-48). 
By (2),

$$
r_{1} \leqq r_{2} \leqq \ldots \leqq r_{n} \rightarrow \infty .
$$

In this paper, $r, \theta$ mean always $r_{n}(z), \theta_{n}(z)$.

Since $\lim u_{n}(z)=0$ uniformly in any compact domain of $F$, the part of $F_{n}$, such that $r_{n}^{\substack{\delta \\ \infty}} \leqq r_{n}(z) \leqq r_{n}(0<\delta<1)$ tends to the ideal boundary of $F$, for $n \rightarrow \infty$. Hence for a given $F_{n}$, we can take $m$ so large that the part $r_{m}^{\delta} \leqq r_{m}(z) \leqq r_{m}$ of $F_{m}$ lies outside of $F_{n}$.

Let $\Delta_{r}$ be the part of $F_{n}-\vec{F}_{0}$, such that $1 \leqq r_{n}(z) \leqq r\left(\leqq r_{n}\right)$ and $C_{r}: r_{n}(z)=r$ $\left(1 \leqq r \leqq r_{n}\right)$ be the niveau curve of $r_{n}(z)$, then by (1),

$$
\int_{C_{r}} d \theta=2 \pi
$$

Let $w(z)$ be one-valued and meromorphic on $F$. We put

$$
m(r, a)=\frac{1}{2 \pi} \int_{c_{r}} \log \frac{1}{[w(z), a]} d \theta,
$$

where

$$
[a, b]=\frac{|a-b|}{\sqrt{\left(1+|a|^{2}\right)\left(1+|b|^{2}\right)}}
$$

Let $n(r, a)$ be the number of zero points of $w(z)-a$ in $\bar{F}_{0}+\Delta_{r}$ and put

$$
\begin{gathered}
N(r, a)=\int_{1}^{r} \frac{n(r, a)}{r} d r-C(a), \quad C(a)=m(1, a) . \\
T_{n}(r, a)=m(r, a)+N(r, a), \\
A(r)=A_{0}+\iint_{\Delta_{r}}\left(\frac{\left|w^{\prime}\right|}{1+|w|^{2}}\right)^{2} r d r d \theta, \quad S(r)=\frac{A(r)}{\pi}, \\
T(r)=\int_{1}^{r} \frac{S(r)}{r} d r,
\end{gathered}
$$

where $w^{\prime}=\frac{d w}{d \zeta}, \zeta=r e^{i \theta}$ and $A_{0}$ is the area of the image of $F_{0}$ by $w=w(z)$ on the $w$-sphere $K$.

Then we shall prove an analogue of Nevanlinna's first fundamental theorem.

THEOREM 1. $\quad T_{n}(r, a)=T_{n}(r) \quad\left(1 \leqq r \leqq r_{n}\right)$.

Though $T_{n}(r)$ is defined for $1 \leqq r \leqq r_{n}$, it is enough for our purpose.

Proof. Considering $w(z)$ as a function of $\zeta=r e^{i 0}$, we have

$$
\begin{aligned}
d m(r, a)-\frac{d m(r, b)}{d r} & =\frac{1}{2 \pi} \int_{c_{r}} \frac{\partial}{\partial r} \log \left|\frac{w-b}{w-a}\right| d \theta \\
& =\frac{1}{2 \pi r} \int_{c_{r}} d \arg \left(\frac{w-b}{w-a}\right)=\frac{n(r, b)-n(r, a)}{r},
\end{aligned}
$$




$$
\frac{d m(r, a)}{d r}+\frac{n(r, a)}{r}=\frac{d m(r, b)}{d r}+\frac{n(r, b)}{r},
$$

hence integrating on $[1, r]$, we have by $(9)$,

$$
T_{n}(r, a)=T_{n}(r, b) .
$$

Let $d \omega(b)$ be the surface element on $K$, then

$$
\begin{aligned}
T_{n}(r, a) & =\frac{1}{\pi} \iint_{\mathrm{K}} T_{n}(r, b) d \omega(b)=\frac{1}{\pi} \iint_{K} m(r, b) d \omega(b) \\
& +\frac{1}{\pi} \iint_{\mathrm{K}} N(r, b) d \omega(b)=\int_{1}^{r} \frac{S(r)}{r} d r+\text { const.. }
\end{aligned}
$$

If we put $r=1$, then we see that const. $=0$, so that

$$
T_{n}(r, a)=T_{n}(r) .
$$

2. To prove that $0 \leqq C(a)=m(1, a) \leqq K$, where $K$ is a constant independent of $a$ and $n$, we shall prove a lemma:

LEMMA. Let $f(z)=u(z)+i v(z)(f(0)=0)$ be regular for $|z| \leqq 1(z=x+i y)$ and $v(x)=0$ for $-1 \leqq x \leqq 1, v(z)>0$ for $y>0,|z| \leqq 1$ and $v(z)=-v(\bar{z})$ for $y<0,|z| \leq 1$.

Then $f(z)$ is schlicht in $|z| \leqq 1 / 7$ 。

Proof. By the hypothesis,

$$
v(z)=v\left(r e^{i 0}\right)=a_{1} r \sin \theta+\sum_{n=2}^{\infty} a_{n} r^{n} \sin n \theta \quad\left(a_{1}>0\right),
$$

where

$$
\begin{aligned}
& a_{1}=\frac{1}{\pi} \int_{-\pi}^{\pi} v\left(e^{i \theta}\right) \sin \theta d \theta=\frac{2}{\pi} \int_{0}^{\pi} v\left(e^{i \theta}\right) \sin \theta d \theta, \\
& a_{n}=\frac{1}{\pi} \int_{-\pi}^{\pi} v\left(e^{i \theta}\right) \sin n \theta d \theta=\frac{2}{\pi} \int_{0}^{\pi} v\left(e^{i \theta}\right) \sin n \theta d \theta .
\end{aligned}
$$

Since $|\sin n \theta| \leqq n|\sin \theta|$,

$$
\left|a_{n}\right| \leqq \frac{2}{\pi} \int_{0}^{\pi} v\left(e^{i \theta}\right)|\sin n \theta| d \theta \leqq \frac{2 n}{\pi} \int_{0}^{\pi} v\left(e^{i \theta}\right) \sin \theta d \theta=n a_{1} .
$$

Since $f(z)=a_{1} z+\sum_{n=2}^{\infty} a_{n} z^{n}$, we have for $\left|z_{1}\right| \leqq r,\left|z_{2}\right| \leqq r \quad(r<1)$,

$$
\begin{aligned}
& \left|\frac{f\left(z_{1}\right)-f\left(z_{2}\right)}{z_{1}-z_{2}}\right|=a_{1}+\sum_{n=2}^{\infty} a_{n}\left(z_{1}^{n-1}+\ldots+z_{2}^{n-1}\right) \geq a_{1}-\sum_{n=2}^{\infty} n\left|a_{n}\right| r^{n-1} \\
& \geq a_{1}\left(1-\sum_{n=2}^{\infty} n^{2} r^{n-1}\right)=a_{1} \frac{1-7 r+6 r^{2}-2 r^{3}}{(1-r)^{3}}>a_{1} \frac{1-7 r}{(1-r)^{3}} \geq 0, \text { if } \quad r \leqq 1 / 7 .
\end{aligned}
$$

Hence $f(z)$ is schlicht in $|z| \leqq 1 / 7$. q.e.d.

We shall prove 
Theorem 2.

$$
0 \leqq C(a)=m(1, a) \leqq K,
$$

where $K$ is a constant, which is independent of $a$ and $n$.

Proof. Let $z_{0}$ be a point of $\Gamma_{0}$ and $U_{0}$ be its neighbourhood, which consists of regular points of $F_{1}$. Then for a suitable $U_{0}$, we can map $U_{0}$ on $|\tau|<1$ conformally, such that $z_{0}$ becomes $\tau=0$ and the part of $U_{0}$, which lies in $F_{1}-F_{0}$ is mapped on the upper half of $|\tau|<1$ and the part of $\Gamma_{0}$, which lies in $U_{0}$ becomes the diameter $L$ of $|\tau|=1$ through $\tau=-1$ and $\tau=1$.

We put $u_{n}(z)=u(\tau)$, then $u(\tau)=0$ on $L, u(\tau)>0$ on the upper half of $\tau<1$, so that $u(\tau)$ can be continued harmonically across $L$ in the lower half of $|\tau|<1$, by putting $u(\tau)=-u(\bar{\tau})$. Hence if we put $f_{n}(z)=-v_{n}(z)+i u_{n}(z)$ $=f(\tau)$, then by the lemma, $f(\tau)$ is schlicht in $|\tau| \leqq 1 / 7$. Hence there exists a constant $R$, such that $f_{n}(z)=-v_{n}(z)+i u_{n}(z)$ is regular and schlicht in $\left|z-z_{0}\right| \leqq R$ for any $z_{0}$ of $\Gamma_{0}$.

Hence by Koebe's distortion theorem, there exists a constant $K_{0}$, such that for any two $z_{1}, z_{2}$ on $\Gamma_{0}$,

$$
\left|f_{n}^{\prime}\left(z_{1}\right)\right| \leqq K_{0}\left|f_{n}^{\prime}\left(z_{2}\right)\right|
$$

Since $u_{n}=0$ on $\Gamma_{l}$, we have

$$
0 \leqq \frac{d v_{n}\left(z_{1}\right)}{d s} \leqq K_{0} \frac{d v_{n}\left(z_{2}\right)}{d s},
$$

where $d s$ is the arc element of $\Gamma_{0}$ and we choose the sense of $\Gamma_{0}$ positive with respect to $F_{0}$. Hence if we put

$$
M_{n}=\operatorname{Max}_{\Gamma_{0}} \frac{d v_{n}(z)}{d s}, \quad m_{n}=\operatorname{Min}_{\Gamma_{0}} \frac{d v_{n}(z)}{d s},
$$

then

$$
M_{n} \leqq K_{0} m_{n}
$$

Now

$$
m(1, a)=\frac{1}{d_{n}} \int_{\Gamma_{0}} \log \frac{1}{[w, a]} \frac{d v_{n}(z)}{d s} d s \leqq \frac{M_{n}}{d_{n}} \int_{\Gamma_{0}} \log \underset{[w, a]}{1} d s \leqq \frac{K_{1} M_{n}}{d_{n}},
$$

where $K_{1}$ is a constant independent of $a$ and $n$.

Since

$$
\boldsymbol{d}_{n}=\int_{\Gamma_{0}} d v_{n} d s \geqq L m_{n},
$$

where $L$ is the length of $\Gamma_{0}$, we have

$$
m(1, a) \leqq \frac{K_{1} M_{n}}{L m_{n}} \leqq \frac{K_{1} K_{0}}{L}=K,
$$

where $K$ is a constant independent of $a$ and $n$. 
3. By means of Theorems 1 and 2, we shall prove

THEOREM 3.2 Let $n(a)$ be the number of zero points of $w(z)-a$ in $F$ and

$$
n_{\mathrm{J}}=\sup _{a} n(a) \text {. }
$$

Let $E$ be the set of $a$, such that $n(a)<n_{0}$, then $E$ is of logarithmic capacity zero.

Proof. First suppose that $n_{0}<\infty$. Then there exists $a_{0}$, such that $n\left(a_{0}\right)=n_{1}$. We take $n$ so large that $w(z)-a_{0}$ has $n_{0}$ zeros in $F_{n}$. Then for any $\delta(0<\delta<1)$, we take $m$ so large that the part of $F_{m}$, such that $r_{n 2}^{\delta} \leqq r_{m}(z) \leqq r_{m}$ lies outside of $F_{n}$, then

$$
T_{m}\left(r_{m}\right) \gtrsim N\left(r_{m}, a_{0}\right) \gtrsim n_{0} \int_{r_{m}}^{r_{m}} \frac{d r}{r}-C(a) \gtrsim n_{0}(1-\delta) \log r_{m}-\mathrm{O}(1) .
$$

Let $E$ be the set of $a$, such that $n(a) \leqq n_{0}-1$ and suppose that cap. $E>0$, then we may assume that $E$ is a bounded closed set. Let $u^{\prime}(w)$ be the equilibrium potential of $E$ :

$$
u(w)=\int_{E} \log \frac{1}{[u, a]} d u(a), \quad \int_{E} d u(a)=1,
$$

such that $u(w)$ is bounded on the $w$-sphere $K$. Then from $m(r, a)+N(r, a)$ $=T_{m}(r)$, we have

$$
\mathrm{O}(1)+\int_{E} N\left(r_{m}, a\right) d \mu(a)=T_{m}\left(r_{m}\right)
$$

so that

$$
T_{m}\left(r_{m}\right) \leqq\left(n_{0}-1\right) \log r_{m}+\mathrm{O}(1)
$$

Since $r_{m} \rightarrow \infty$, we have from (1), (2),

$$
n_{0}(1-\delta) \leqq n_{0}-1 \text {, }
$$

which is impossible, if $\delta<1 / n$. . Hence cap. $E=0$.

If $n_{0}=\infty$, then for any $N>0$, there exists $a_{3}$, such that $n\left(a_{0}\right)>N$, then the set of $a$, such that $n(a) \leqq N-1$ is of logarithmic capacity zero. Since $N$ is arbitrary, the set of $a$, such that $n(a)<\infty$ is of logarithmic capacity zero.

Remark. Let $\Phi$ be the Riemann surface of the inverse function $z=z(w)$ of $w(z)$ spread over the $w$-sphere $K$. If $n_{0}<\infty$, then the set of $a$, such that $n(a)=n_{0}$ is an open set, so that $\Phi$ consists of $n_{0}$ sheets and the projection of singular points of $z(w)$ on $K$ is a closed set of logarithmic capacity zero.

From the above proof, we have easily

2) Y. Nagai: On the behaviour of the boundary of Riemann surfaces, II. Proc. Japan Acad. 26 (1950). Z. Yûjôbô: On the Riemann surfaces, no Green's function of which exists. Mathematica Japonicae. II, No. 2(1951). M. Tsuji: Some metrical theorems on Fuchsian groups. Kodai Math. Seminar Reports. Nos. 4-5 (1950). A. Mori: On Riemann surfaces on which no bounded harmonic function exists. Journ. Math. Soc. Japan. 3 (1951). 
THEOREM 4. If $n_{0}=\sup _{a} n(a)=\infty$, then $w(z)$ takes any value infinitely often, except a set of logarithmic capacity zero and

$$
\lim _{n \rightarrow \infty} \frac{T_{n}\left(r_{n}\right)}{\log r_{n}}=\infty
$$

Conversely, if this condition is satisfied, then $w(z)$ takes any valuse infinitely oftern, except a set of logarithmic capacity zero.

\section{$\S 2$}

1. Let $\Phi$ be the Riemann surface of the inverse function $z=z(w)$ of $w(z)$ spread over the $w$-plane and $w_{0}$ be its regular point. We continue $z(w)$ along a half-line $L(\varphi): \arg \left(w-w_{0}\right)=\varphi$ till we meet a singular point of $z(w)$. Then we obtain the Mittag-Leffler's principal star region $H\left(w_{0}\right)$. Let $E$ be the set of $\varphi$, such that $L(\varphi)$ meets a singular point of $z(w)$ at a finite distance. Then

TheOREM $5 .^{31} \quad E$ is of measure zero.

This is an extension of Gross' theorem."

Proof, Let $H_{R}\left(w_{0}\right)$ be the part of $H\left(w_{0}\right)$, which lies in $\left|w-w_{0}\right|<R$ and $E_{R}$ be the sei of $\varphi$, such that $L(\varphi)$ meets a singular point of $z(w)$ in $\left|w-w_{0}\right|<R$. Let $F_{R}$ be the image of $H_{R}\left(w_{0}\right)$ on $F$ and $C_{r}(R)$ be the part of $C_{r}$ contained in $F_{s 2}$ and $s(r)$ be the length of its image in $H_{R}\left(w_{0}\right)$, then writing $w^{\prime}=\frac{d w}{d \zeta}$, $\zeta=r e^{i s}$, we have

$$
s(r)^{2}=\left(\int_{C_{r}(R)}\left|w^{\prime}\right| r d \theta\right)^{2} \leqq 2 \pi r \int_{C_{r}(R)}\left|w^{\prime}\right|^{2} r d \theta=2 \pi r \frac{d A(r)}{d r},
$$

where $A(r)$ is the area of the image of $\Delta_{r} \cdot F_{R}$ in $H_{B}\left(w_{0}\right)$. Hence

$$
\int_{\bar{r}_{r_{n}}}^{r_{n}} \frac{s(r)^{2}}{r} d r \leqq 2 \pi A\left(r_{n}\right) \leqq 2 \pi^{2} R^{2}
$$

Hence if we put $\operatorname{Min} s(r)=s_{n}$, then

$$
s_{n}^{2} \log r_{n} \leqq 4 \pi^{2} R^{2} \text { 。 }
$$

Since $r_{n} \rightarrow \infty$, we have $s_{n} \rightarrow 0$, so that $m E_{R}=0$. Since $R$ is arbitrary, we have $m E=0$.

2. Let a Riemann surface $F$ be spread over the $z$-shpere $K$. If there exists a sequence of compact Riemann surfaces $F_{n} \rightarrow F$, such that $L_{n} /\left|F_{n}\right| \rightarrow 0(n \rightarrow \infty)$,

3) K. Noshiro: Open Riemann surface with null boundary. Nagoya Math. Journ. 3 (1951). Z. Yûjôbô. 1. c. 2).

4) W. Gross: Über die Singularitäten analytischer Funktionen. Monatshefte f. Math. u. Phys. 29 (1918), 
then $F$ is called regularly exhaustible in Ahlfors' sense, where $L_{n}$ is the length of the boundary of $F_{n}$ and $\left|F_{n}\right|$ is its area measured on $K$.

THEOREм 6.5 The Riemann surface $\Phi$ of the inverse function $z(w)$ of $w(z)$ is regularly exhaustible in Ahifors' sense.

Proof. Writing $w^{\prime}=\frac{d w}{d \zeta}, \zeta=r e^{i \emptyset}$, we put as in $\S 1$,

$$
\begin{gathered}
A(r)=A_{0}+\iint_{\Delta r}\left(\frac{\left|w^{\prime}\right|}{1+|w|^{2}}\right)^{2} r d r d \theta, \\
L(r)=\int_{c_{r}} \frac{\left|w^{\prime}\right|}{1+|w|^{2}} r d \theta_{0}
\end{gathered}
$$

Then

$$
L(r)^{2} \leqq 2 \pi r \frac{d A(r)}{d r}
$$

(i) First suppose that $A\left(r_{n}\right) \rightarrow \infty \quad(n \rightarrow \infty)$ and suppose that

$$
L(r)>(A(r))^{3 / 4}
$$

for any $r$, such that $\sqrt{r_{n}} \cong r \leqq r_{n}$, then

$$
\int_{V_{\overline{r n}}}^{r n} \frac{d r}{r} \leqq 2 \pi \int_{1}^{r_{n}} \frac{d A(r)}{(A(r))^{3 / 2}} \leqq \frac{4 \pi}{A_{0}}
$$

Since $\int_{\gamma_{\overline{r_{n}}}}^{r_{n}} \frac{d r}{r}=\frac{1}{2} \log r_{n} \rightarrow \infty$, this is absurd, hence there exists $\tau_{n}\left(\sqrt{r_{n}} \leqq \tau_{n} \leqq r_{n}\right)$, such that

$$
L\left(\tau_{n}\right) \leqq\left(A\left(\tau_{n}\right)\right)^{3 / 4}
$$

Since $A\left(\tau_{n}\right) \rightarrow \infty$ with $A\left(r_{n}\right) \rightarrow \infty$, we have

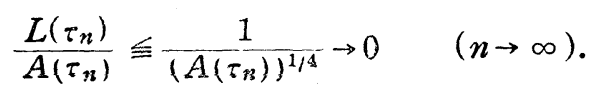

(ii) If $A\left(r_{n}\right) \leqq K(n \rightarrow \infty)$, then

$$
\int_{r_{\overline{r_{n}}}}^{r_{n}} \frac{L(r)^{2}}{r} d r \leqq 2 \pi A\left(r_{n}\right) \leqq 2 \pi K
$$

so that there exists $\tau_{n}\left(\sqrt{r_{n}} \leqq \tau_{n} \leqq r_{n}\right)$, such that $L\left(\tau_{n}\right) \rightarrow 0$, hence

$$
\frac{L\left(\tau_{n}\right)}{A\left(\tau_{n}\right)} \rightarrow 0 \quad(n \rightarrow \infty) \text {. }
$$

Hence our theorem is proved.

\section{§3}

1. As an application of Theorem 6, we shall prove an extension of Myrberg's

5) K. Noshiro: 1. c. 3), 
theorem. Let $F$ be a closed Riemann surface of genus $p \geqslant 2$, spread over the $z$-sphere $K$. We make $F$ become a surface of planar character by cutting along $p$ disjoint ring cuts $C_{i}(i=1,2, \ldots, p)$, and let $F_{\mathrm{c}}$ be the resulting surface. We take infinitely many same samples as $F_{0}$ and connect them along the opposite shores of $C_{i}$ as in the well known way, then we obtain a covering surface $F^{(\infty)}$ of $F$, which is of planar character. Hence by Koebe's theorem, we can map $F^{(\infty)}$ on a schlicht domain $D$ on the $\zeta$-plane. The boundary $E$ of $D$ is a bounded perfect set, which is the singular set of a certain linear group of Schottky type. Myrberg ${ }^{6)}$ proved that $E$ is of positive logarithmic capacity, hence $F^{(\infty)}$ is of positive boundary.

We shall generalize this Myrberg's theorem as follows.

Instead of cutting $F$ along $p$ ring cuts, we cut $F$ along $q(1 \leqq q \leqq p)$ ring cuts $C_{i}(i=1,2, \ldots, q)$ and let $F_{0}$ be the resulting surface. We take infinitely many same samples as $F_{0}$ and connect them along the opposite shores of $C_{i}(i=1$, $2, \ldots, q)$, then we obtain a covering surface $F_{(q)}^{(\infty)}$ of $F$, which is of infinite genus, if $q<p$.

2. We shall prove

THEOREM 7. $F_{(1)}^{(\infty)}$ is of null boundary, while if $q \geq 2, F_{(q)}^{(\infty)}$ is of positive boundary and there exists $a$ non-constant bounded harmonic function $u(z)$ on $F_{(q)}^{(\infty)}$, whose Dirichlet integral $D[u]$ on $F_{(q)}^{(x)}$ is finite.

Proof. (i). First we shall prove that $F_{(1)}^{(x)}$ is of null boundary. We cut $F$ along $C_{1}=C$ and let $F_{0}$ be the resulting surface. We take infinitely many same samples as $F_{0}$ :

$$
\begin{aligned}
& F_{1}^{\prime}, F_{2}^{\prime}, \ldots, F_{n}^{\prime}, \ldots \\
& F_{1}^{\prime \prime}, F_{2}^{\prime \prime}, \ldots, F_{n}^{\prime \prime}, \ldots
\end{aligned}
$$

Let $C^{+}, C^{-}$be the both shores of $C$. When we consider them belong to the boundary of $F_{n}^{\prime}$, we denote them by $\left(C^{+}\right)_{n}^{\prime},\left(C^{-}\right)_{n}^{\prime}$.

Similarly we define $\left(C^{+}\right)_{n}^{\prime \prime},\left(C^{-}\right)_{n}^{\prime \prime}$ for $F_{n}^{\prime \prime}$.

We connect $\left\{F_{n}^{\prime}\right\},\left\{F_{n}^{\prime \prime}\right\}$ as follows.

We identify $C^{+}$of $F_{0}$ with $\left(C^{-}\right)_{1}^{\prime}$ of $F_{1}^{\prime},\left(C^{+}\right)_{1}^{\prime}$ of $F_{1}^{\prime}$ with $\left(C^{-}\right)_{2}^{\prime}$ of $F_{2}^{\prime}$ and so on. We identify $C^{-}$of $F_{0}$ with $\left(C^{+}\right)_{1}^{\prime \prime}$ of $F_{1}^{\prime \prime},\left(C^{-}\right)_{1}^{\prime \prime}$ of $F_{1}^{\prime \prime}$ with $\left(C^{+}\right)_{2}^{\prime \prime}$ of $F_{2}^{\prime \prime}$ and so on and put

$$
F_{n}=F_{0}+\sum_{\nu=1}^{n} F_{\nu}^{\prime}+\sum_{\nu=1}^{n} F_{\nu}^{\prime \prime}, \quad F_{n}-F_{n-1}=F_{n}^{\prime}+F_{n}^{\prime \prime}
$$

We take a circular disc $\Delta_{0}$ in $F_{0}$ and let $\Gamma_{0}$ be its boundary.

6) P. J. Myrberg: Die Kapazität der singulären Menge der linearen Gruppen. Ann. Acad. Fenn. Ser. A. Math.-Phys. 10 (1941). M. 'Tsuji: On the uniformization of an algebraic function of genus $p \geq 2$. Tohoku Math. Journ. 3 (1951). 
Then

$$
\left.\Delta_{0} \subset F_{0} \subset F_{1} \subset \ldots \subset F_{n \rightarrow} F_{(1)}^{(\infty)}\right)
$$

The boundary $\Gamma_{n}$ of $F_{n}$ is

$$
\Gamma_{n}=\left(C^{+}\right)_{n}^{\prime}+\left(C^{-}\right)_{n}^{\prime \prime}
$$

Let $u_{n}^{(0)}(z)$ be the harmonic measure of $\Gamma_{n}$ with respect to $F_{n}-\bar{\Delta}_{0}$ and let $v_{n}^{(0)}(z)$ be its conjugate harmonic function and put

$$
d_{n}^{(0)}=\int_{\Gamma_{0}} d v_{n}^{(0)}(z), \quad \mu_{n}^{(0)}=2 \pi / d_{n}^{(0)} .
$$

Let $u_{n}^{\prime}(z)$ be the harmonic measure of $\left(C^{+}\right)_{n}^{\prime}$ with respect to $F_{n}^{\prime}$, such that $u_{n}^{\prime}(z)$ $=0$ on $\left(C^{-}\right)_{n}^{\prime}, u_{n}^{\prime}(z)=1$ on $\left(C^{+}\right)_{n}^{\prime}$ and let $v_{n}^{\prime}(z)$ be its conjugate harmonic function.

Let $u_{n}^{\prime \prime}(z)$ be the harmonic measure of $\left(C^{-}\right)_{n}^{\prime \prime}$ with respect to $F_{n}^{\prime \prime}$, such that $u_{n}^{\prime \prime}(z)=0$ on $\left(C^{+}\right)_{n}^{\prime \prime}, u_{n}^{\prime \prime}(z)=1$ on $\left(C^{-}\right)_{n}^{\prime \prime}$. We put

$$
d_{n}=\int_{\left(C^{-}\right)_{n}^{\prime}} d v_{n}^{\prime}(z)+\int_{\left(C^{+}\right)_{n}^{\prime \prime}} d v_{n}^{\prime \prime}(z), \quad \mu_{n}=2 \pi / d_{n} .
$$

Then as Noshiro ${ }^{7)}$ proved,

$$
\mu_{n}^{(0)} \geq \mu_{0}^{(0)}+\mu_{1}+\ldots+\mu_{n} .
$$

Since $\mu_{n} \supseteqq$ const. $=a>0$, we have $\lim _{n \rightarrow \infty} \mu_{n}^{(0)}=\infty$, so that $\lim _{n \rightarrow \infty} d_{n}^{(0)}=0$, hence $F_{(1)}^{(\infty)}$ is of null boundary.

(ii) Next we shall prove that $F_{(q)}^{(\infty)}(q \geqslant 2)$ is of positive boundary. Suppose that $F_{(q)}^{(\infty)}$ is of null boundary, then by Theorem $6, F_{(q)}^{(\infty)}$ is regularly exhaustible in Ahlfor's sense, so that there exists a sequence of compact Riemann surfaces: $F_{1} \subset F_{2} \subset \ldots \subset F_{n} \rightarrow F_{(q)}^{(\infty)}$, such that

$$
\frac{L_{n}}{S_{n}} \rightarrow 0, \quad(n \rightarrow \infty),
$$

where $L_{n}$ is the length of the boundary $\Gamma_{n}$ of $F_{n}$. measured on the $z$-sphere $K$ and

$$
S_{n}=\frac{\left|F_{n}\right|}{|F|},
$$

where $\left|F_{n}\right|,|F|$ are the spherical areas of $F_{n}$ and $F$ respectively.

As seen from the proof of Theorem $6, \Gamma_{n}$ is the niveau curve of a harmonic measure, so that $\Gamma_{n}$ consists of a finite number $\nu_{n}$ of disjoint closed curves, which are not homotop null, hence the length of each curve is $\vec{z} a>0$, where $a$ is a constant, which depends on $F$ only. Hence

$$
L_{n} \geqslant a \nu_{n} \text {. }
$$

i) K. Noshiro: 1, c. 3). 
We denote the Euler's characteristic of $F_{n}$ by $\rho_{n}$.

Let $C_{i}(i=q+1, \ldots, p)$ be covered $\mu_{i}^{(n)}$ times by $F_{n}$, then we see easily that

$$
\rho_{n} \leqq 2\left(\mu_{q+1}^{(n)}+\ldots+\mu_{p}^{(n)}\right)+\nu_{n} \leqq 2\left(\mu_{q+1}^{(n)}+\ldots+\mu_{p}^{(n)}\right)+L_{n} / a .
$$

Now by Ahlfors' second covering theorem, ${ }^{8)}$

$$
\mu_{i}^{(n)} \leqq S_{n}+h L_{n} \text {, }
$$

where $h$ is a constant, which depends on $F$ only, so that

$$
\rho_{n} \leqq 2(p-q) S_{n}+h L_{n}
$$

with a suitable $h$.

Since $\rho_{0}=2(p-1)$ is the Euler's characteristic of $F$, we have by Ahlfors' fundamental theorem on covering surfaces, ${ }^{9)}$

$$
\rho_{n} \text { 를 } 2(p-1) S_{n}-h L_{n},
$$

so that by $(6)$,

$$
2(q-1) S_{n} \leqq h L_{n},
$$

which contradicts (1), if $q \geqslant 2$. Hence $F_{(q)}^{(\infty)}(q \geqslant 2)$ is of positive boundary.

Next we shall prove that there exists a non-constant bounded harmonic function $u(z)$ on $F_{(q)}^{(x)}$, whose Dirichlet integral $D[u]$ is finite.

We take off $F_{0}$ from $F_{(q)}^{(\infty)}$, then there remains $2 q$ connected surfaces $\Phi_{i}^{+}, \Phi_{i}^{-}$ $(i=1,2, \ldots, q)$, where $\Phi_{i}^{+}$abutts on $F_{0}$ along $C_{i}^{+}$and $\Phi_{i}^{-}$abutts on $F_{0}$ along $C_{i}^{-}$.

We exhaust $\Phi_{1}^{+}=\Phi$ by a sequence of compact Riemann surfaces: $\Phi_{1} \subset \Phi_{2}$ $\subset \ldots \subset \Phi_{n} \rightarrow \emptyset$, where $C_{1}^{+}+\Gamma_{n}$ is the boundary of $\Phi_{n}$. Let $u_{n}(z)$ be the harmonic measure of $\Gamma_{n}$ with respect to $\Phi_{n}$ and let $v_{n}(z)$ be its conjugate harmonic function and put

$$
d_{n}=\int_{c_{i}^{+}} d v_{n}(z)
$$

Since $u_{n}(z)$ decreases with $n, d_{n}$ decreases with $n$.

From the above proof, we see that

$$
\lim _{n \rightarrow \infty} d_{n}>0 \text {. }
$$

Let

$$
\lim _{n \rightarrow \infty} u_{n}(z)=u(z)
$$

and $v(z)$ be its conjugate harmonic function and put

8) L. Ahlfors: Zur Theorie der Überlagerungsfächen. Acta Math. 65 (1935).

9) L. Ahlfors: 1. c. 8). 


$$
d=\int_{c_{1}^{+}} d v(z)
$$

then $\lim _{n \rightarrow \infty} d_{n}=d>0$, so that $u(z) \neq$ const., hence $u(z)=0$ on $C_{1}^{+}, 0<u(z)<1$ in $\emptyset$. Let $D[u]$ be the Dirichlet integral of $u(z)$ on $\emptyset$, then

$$
0<D[u] \leqq d<\infty .
$$

Hence there exists a non-constant bounded harmonic function on $\emptyset$, which vanishes on $C_{1}^{+}$and whose Dirichlet integral is finite. Similarly there exists a similar harmonic function on $\Phi_{i}^{+}, \Phi_{i}^{-}$.

Hence as proved by R. Nevanlinna ${ }^{10)}$ and Bader and Pareau, ${ }^{11)}$ there exists a non-constant bounded harrnonic function on $F_{(q)}^{(\infty)}$, whose Dirichlet integral is finite.

Remark. By Sario's theorem, ${ }^{12}$ there exists no non-constant one-valued regular function on $F_{(q)}^{(\infty)}$, whose Dirichlet integral is finite.

\section{$\S 4$}

Let $w(z)$ be one valued and meromorphic on $F$ and $\Phi$ be the Riemann surface of the inverse function $z(w)$ of $w(z)$ spread over the $w$-plane and $\Phi^{(\rho)}$ be a connected piece of $\emptyset$, which lies above $\left|w-w_{0}\right|<\rho$ and $F^{(\rho)}$ be its image on $F$. We assume that $F^{(p)}$ is non-compact.

With the same notations as $\S 1$ we put

$$
\Delta_{r}^{(\rho)}=\Delta_{r} \cdot F^{(\rho)}, \quad F_{n}^{(\rho)}=F_{n} \cdot F^{(\rho)}, \quad C_{r}^{(\rho)}=C_{r} \cdot F^{(\rho)} .
$$

For the sake of brevity we assume that $w_{0}=0$.

To define $m(r, a)$, we introduce a metric $(a, b)$ in $|w|<\rho$ as follows. ${ }^{13)}$

For $|a|<\rho$, we put

$$
(a, 0)=\frac{2 \rho|a|}{\rho^{2}+|a|^{2}}
$$

Let $U_{a}(w)=\frac{\rho^{2}(w-a)}{\rho^{2}-\bar{a} w}$, then for $|a|<\rho,|b|<\rho$, we define $(a, b)$ by

$$
(a, b)=\left(U_{a}(b), 0\right)=\frac{2 \rho|b-a| /\left|\rho^{2}-\bar{a} b\right|}{1+\rho^{2}|b-a|^{2} /\left|\rho^{2}-\bar{a} b\right|^{2}} .
$$

By this metric, we put

10) R. Nevanlinna: Über der Existenz von beschränkten Potentialfunktionen auf Flächen von unendlichem Geschlecht. Math. Zeits. 52 (1950).

11) R. Bader and M. Pareau: Domaines non-compacts et classification des surfaces de Riemann. C.R. 232 (1951). A. Mori: On the existence of harmonic functions on a Riemann surface. Journ. Fac. Sci. Tokyo Univ. Section I, Vol. VI, Part 4 (1951).

12) L. Sario: Über Riemannsche Fläche mit hetbarem Rand. Ann. Acad. Fenn. A. I. 50 (1948).

13) M. Tsuji: On a regular function which is of constant absolute value on the boundary of an infinite domain. Tohoku Math. Journ. 3 (1951). 


$$
m(r, a)=\frac{1}{2 \pi} \int_{c_{r}^{(p)}} \log \frac{1}{(w(z), a)} d \theta
$$

We use the same notations as $\S 1$, since no confusion occurs.

Let $n(r, a)$ be the number of zero points of $w(z)-a$ in $\bar{F}_{0}^{(p)}+\Delta_{r}^{(p)}$ and put

$$
N(r, a)=\int_{1}^{r} \frac{n(r, a)}{r} d r-C(a), \quad C(a)=m(1, a) .
$$

Then as Theorem 2,

$$
0 \leqq C(a) \leqq K,
$$

where $K$ is a constant independent of $a$ and $n$. We put

$$
\begin{gathered}
T_{n}(r, a)=m(r, a)+N(r, a), \\
A(r)=A_{0}+\iint_{\left.\Delta_{\rho}^{(}\right)}\left(\frac{\left|w^{\prime}\right|}{1+|w|^{2}}\right)^{2} r d r d \theta, \quad S(r)=\frac{A(r)}{\sigma(\rho)},
\end{gathered}
$$

where $A_{0}$ is the area of the image of $F_{0}^{(\rho)}$ on the $w$-sphere $K$ by $w=w(z)$, $w^{\prime}=\frac{d w}{d \zeta}, \quad \zeta=r e^{i \theta}$ and $\sigma(\rho)=\frac{\pi \rho^{2}}{1+\rho^{2}}$ is the area of the projection of $|w| \leqq \rho$ on $K$.

$$
\begin{gathered}
T_{n}(r)=\int_{1}^{r} \frac{S(r)}{r} d r \\
L(r)=\int_{c_{r}^{(\rho)}} \frac{\left|w^{\prime}\right|}{1+|w|^{2}} r d \theta
\end{gathered}
$$

Then similarly as my former paper, ${ }^{14)}$ we have

THEOREM 8. $T_{n}(r, a)=T_{n}(r)+\mathrm{O}(\not(r)), \quad\left(1 \leqq r \leqq r_{n}\right)$, where

For $|a| \leqq \rho_{1}<\rho$,

$$
\Phi(r)=\int_{1}^{r} \frac{L(r)}{r} d r
$$

$$
|\mathrm{O}(\boldsymbol{D}(\boldsymbol{r}))| \leqq K \Phi(r)
$$

where $K$ is a constant, which depends $\rho_{1}$ on only.

THEOREM 9. For any $\delta(0<\delta<1)$, there exists $\tau_{n}\left(r_{n}^{1-\delta} \leqq \tau_{n} \leqq r_{n}\right)\left(n\right.$ 포 $\left.n_{0}\right)$, such that

$$
\emptyset\left(\tau_{n}\right) \leqq \sqrt{T_{n}\left(\tau_{n}\right)} \log T_{n}\left(\tau_{n}\right) .
$$

Hence for any $\varepsilon>0$,

$$
(1-\varepsilon) T_{n}\left(\tau_{n}\right) \leqq T_{n}\left(\tau_{n}, a\right) \leqq(1+\varepsilon) T_{n}\left(\tau_{n}\right) \quad\left(n \geqslant n_{1}\right) .
$$

11) M. Tsuji: 1. c. 13). 
Proof. We follow Dinghas. ${ }^{15)}$ By (10), (8),

so that

$$
\begin{gathered}
L(r)^{2} \leqq 2 \pi r \hat{\int}_{C_{r}^{(p)}}\left(\frac{\left|w^{\prime}\right|}{1+|w|^{2}}\right)^{2} r d \theta=2 \pi r \frac{d A(r)}{d r}, \\
L(r) \leq \sqrt{2 \pi} \sqrt{\frac{A^{\prime}(r)}{r}}, \\
r \quad
\end{gathered}
$$

$$
\Phi(r) \leqq \sqrt{2} \pi \int_{1}^{r} \sqrt{\frac{A^{\prime}(r)}{r}} d r
$$

Hence

$$
(\Phi(r))^{2} \leqq 2 \pi \int_{1}^{r} \frac{d r}{r} \int_{1}^{r} A^{\prime}(r) d r=2 \pi \sigma(p) r \log r \cdot T_{n}^{\prime}(r) .
$$

Suppose that

$$
\Phi(r)>\sqrt{ } T_{n}(r) \log T_{n}(r)
$$

for any $r$, such that $r_{n}^{1-\delta} \leqq r \leqq r_{n}$, then

$$
\begin{aligned}
& \int_{r_{10}^{1-\delta}}^{r_{n}} \frac{d r}{r \log r} \leqq 2 \pi \sigma(\rho) \int_{r_{n}^{1-\delta}}^{r_{n}} \frac{d T_{n}(\boldsymbol{r})}{T_{n}(\boldsymbol{r}) \log ^{2} T_{n}(\boldsymbol{r})} \leqq 2 \pi \sigma(\rho) \frac{1}{\log T_{n}\left(r_{n}^{1-\delta}\right)} \rightarrow 0 \\
& (n \rightarrow \infty) \text {. }
\end{aligned}
$$

Since $\int_{r_{n}^{1-\delta}}^{r_{n}} \frac{d r}{r \log r}=\log \frac{1}{1-\delta}$, this is absurd, hence there exists $:_{n}\left(r_{1 !}^{1-\delta} \leqq \tau_{\eta} \leqq r_{n}\right)$ $\left(n \geq n_{0}\right)$, such that

$$
\emptyset\left(\tau_{n}\right) \leqq \sqrt{T_{n}\left(\tau_{n}\right)} \log T_{n}\left(\tau_{n}\right) .
$$

THEOREM 10.16) Under the same condition as Theorem 8 , let $n(a)$ be the number of zero points of $w(z)-a(|a|<\rho)$ in $F^{(\rho)}$ and

$$
r_{0}=\sup _{a} n(a) \text {. }
$$

Let $E$ be the set of a, such that $n(a)<n_{0}$. Then $E$ is of logarithmic capacity zero.

Proof. (i) First suppose that $n_{0}<x$. Then there exists $a_{0}$, such that $n\left(a_{0}\right)=n_{0}$ and let $w(z)-a_{0}$ has $n_{0}$ zeros in $F_{n}^{(\rho)}$.

We take $m$ so large that the part: $\gamma_{m}^{\delta} \leqq r_{m}(z) \leqq r_{m}$ of $F_{\eta ;}^{(p)}$ lies outside of $F_{n}^{(\rho)}$. By Theorem 9, there exists $\tau_{m}\left(r_{m}^{1-\delta} \leqq \tau_{m} \leqq \gamma_{m}\right)$, such that

$$
\begin{aligned}
(1+\delta) T_{m}\left(\tau_{m}\right) & \Rightarrow T_{m}\left(\tau_{m}, a_{0}\right) \gg N\left(\tau_{m}, a_{0}\right) \geqslant n_{0} \int_{r_{m}^{\delta}}^{r_{m}^{1-\delta}} \frac{d r}{r}-C(a) \\
& =n_{0}(1-2 \delta) \log r_{m}-\mathrm{O}(1) .
\end{aligned}
$$

Let $E$ be the set of $a$, such that $n(a) \leqq n_{0}-1$ and suppose that cap. $E>0$.

15) A. Dinghas: Eine Bemerkung zur Ahlforsschen Theorie der Überlagerungsflächen. Math. Zeits. 44 (1936).

16) Y. Nagai: 1. c. 2). Z. Yûjòbô: 1. c. 2). M. Tsuji: 1. c. 2). A. Mori: 1. c. 2). 
Then we may assume that $E$ is a closed set contained in $|w| \leqq \rho_{1}<\rho$. Then similarly as Theorem 3 , we have

$$
(1-\delta) T_{m}\left(\tau_{m}\right) \leqq\left(n_{0}-1\right) \log r_{m}+\mathrm{O}(1) \text {. }
$$

Hence from (1), (2),

$$
\frac{n_{0}(1-2 \delta)}{1+\delta} \leqq \frac{n_{0}-1}{1-\delta}
$$

which is impossible, if $\delta$ is sufficiently small. Hence cap. $E=0$. If $n_{0}=\infty$, then we can prove similarly as Theorem 3 , that the set $E$ of $a$, such that $n(a)$ $<\infty$ is of logarithmic capacity zero.

Mathematical Institute,

Tokyo University 